\title{
Osteogenic Growth Factors Complex from Human Mesenchymal Stem Cells, Cultivated by using Robotic Station
}

\author{
Mikhail Khotin ${ }^{1}$, Svetlana Aleksandrova ${ }^{1}$, Liubov Pokrovskaya ${ }^{2}$, Sergey Nadezhdin ${ }^{3}$, Ekaterina \\ Zubareva $^{3}$, Stanislav Vasilyev ${ }^{4}$, Renata Savchenko ${ }^{4}$, Miralda Blinova ${ }^{1}$, Natalia Mikhailova ${ }^{1}$ \\ ${ }^{1}$ Institute of Cytology of the Russian Academy of Science \\ Tikhoretsky ave. 4, 194064 St-Petersburg, Russia \\ h_mg@mail.ru, alekssvet2205@gmail.com; mira.blinova@mail.ru; natmik@mail.ru \\ ${ }^{2}$ National Research Tomsk State University \\ Lenin ave, 634050, 36, Tomsk, Russia \\ pokrovskayal@ect-center.com; \\ ${ }^{3}$ Belgorod National Research University \\ Pobedy St. 85, 308015, Belgorod, Russia \\ nadezhdin@bsu.edu.ru; zubareva@bsu.edu.ru; \\ ${ }^{4}$ Research Institute of Medical Genetics, Tomsk National Research Medical Center, \\ Nab.r. Ushaiki, 10, Tomsk, 634050, Russia \\ stanislav.vasilyev@medgenetics.ru; renata.savchenko@medgenetics.ru
}

Keywords: Bone tissue engineering, Human bone marrow mesenchymal stem cells, Secretome, Robotic station.

\section{Extended Abstract}

The cell technologies development is based on evidence of the safety and efficacy of different types of stem cells, even after in vitro cultivation. The different types of cell products are considered in bone tissue restoration. A number of studies have shown that the factors produced by cells (secretome) during differentiation, may be a signal for the stem cells to differentiate. Therefore a therapeutic effect can be achieved by introducing that factors. At present, there is an important task in developing of technology that allows to obtain secretome concentrate from stem cells (in sufficient quantities and of the required quality).

The aim of this work was to design and standardize the protocol for obtaining a concentrate of conditioned medium from human mesenchymal stem cells after their differentiation in the osteogenic direction.

Non-transformed cell line FetMSC (Vertebrate Cell Culture Collection, Institute of Cytology, Russian Academy of Sciences), obtained from stromal cells of a 5-6 week embryo`s bone marrow, was used as a line of human mesenchymal stem cells (MSCs).

A robotic station Compact Select T (Sartorius), providing of standardization of all processes of working with cells was used to cultivate and differentiate the cells. The work was carried out under conditions of experimental production of biomedical cell products under clean-room conditions. The Compact Select system allowed mass cultivation of 70 and more flasks T-175 simultaneously with the guarantee of full repeatability of the protocol. This approach allowed to adapt technology to industrial. Cultivation performed under aseptic conditions at controlled temperature parameters $\left(37^{\circ} \mathrm{C}\right)$, humidity (98\%), and composition of the gaseous medium $\left(5 \% \mathrm{CO}_{2}\right)$. FetMSC were cultured in DMEM/F12 (Rosmedbio, Russia) containing 10\% fetal bovine serum (HyClone, CШA) and 1\% glutamine (Lonza, Switzerland). Then the mix containing $10 \mathrm{mM}$ sodium $\beta$-glycerophosphate (\# G5422, 2013, Sigma, USA), $100 \mathrm{nM}$ dexamethasone (\# D2915, Sigma, USA) and $50 \mu \mathrm{g} / \mathrm{ml}$ ascorbic acid (\# Cat 102890, ICN Biomedical Inc., USA) was added to cultivation medium to differentiate the cells. Osteoinductive medium was replaced of a serum-free growth medium. Then, in an automated mode, the conditioned medium was collected in one container, centrifuged at $800 \mathrm{~g}$ during 10 minutes and the resulting supernatant was passed through a $0.22 \mu \mathrm{m}$ filter for sterilization.

In the process of cultivation the monitoring of cellular parameters was carried out in real time: the morphology of the cells, their viability and quantity. For this, the built-in Compact Select systems were used: the cell counter ViCell (Beckman) and the cell analyzer IncuCyte (Sartorius). At the end of the differentiation protocol cells were analyzed by real-time PCR for the expression of the transcription factor genes RUNX2 and YAP1, appearing during early stages of osteogenesis.

For PCR RNA from the samples was extracted using "Lira" reagent (Biolabmix, Russia), which contains phenol and guanidine thiocyanate. Reverse transcription was carried out using OT M-MuLV-RH reagent kit (Biolabmix, Russia). 
Specific PCR-products were obtained using self-designed primers to PPIA (reference gene), RUNX2 and YAP1 genes. Real-time PCR was conducted using AriaMx amplifier (Agilent Technologies, USA).

The conditioned medium was concentrated by ultrafiltration in a tangential flow using a Vivaflow ${ }^{\circledR} 200$ system (Sartorius) using a membrane module with a cut-off threshold for molecules with a molecular mass of $10 \mathrm{kDa}$. The resulting concentrate was dialyzed using the Vivaflow ${ }^{\circledR} 200$ system (Sartorius), adding deionized water to the sample/diafiltration tank. To prepare the growth factors concentrate, the conditioned medium concentrate was dried in a Rotavapor ${ }^{\circledR}$ R-210 vacuum rotary evaporator (Buchi), collected aseptically, placed in a microcentrifuge tube with a screw cap and a sealing ring in the freezer at $-20{ }^{\circ} \mathrm{C}$ ( or $-80{ }^{\circ} \mathrm{C}$ for a longer time).

FetMSC cell line was decryoconserved and processed in the robotic station up to operating cell mass in the amount of $5^{*} 10^{8}$ cells. Morphology, doubling times and viability shown high standard indicators. A new algorithm for gaiding of robotic station was created for special cultivation conditions. After replacing the growth medium with the differentiating medium and further cultivation in serum-free medium the cell mass reached a number of $7 * 10^{8}$ cells. After collecting the conditioned medium, an assessment was made of the state of the cells remaining in the culture flasks, which showed the viability of $99 \%$ of the cell mass. That cells were analyzed by real-time PCR for reveling of transcription factor genes RUNX2 and YAP1 expression. It was shown that after cultivation protocol the expression was in two times higher than in the undifferentiated cells.

Under aseptic conditions $1000 \mathrm{ml}$ of conditioned medium were collected in a sterile glass container and purificate from cellular elements. Transportation and storage were carried out at $4{ }^{\circ} \mathrm{C}$. The resulting medium was used to obtain a concentrate of growth factors of cellular origin.

Thus, the technology of obtaining the conditioned medium from differentiated MSCs was developed and tested under conditions of pilot production and with the use of robotic technologies. The developed technology can be used as a matrix for obtaining therapeutic products based on the secretome of cultured stem or differentiated human cells of the medical grade level.

The investigation was funded by the Ministry of Science and Higher Education of the Russian Federation, agreement № 14.575.21.0164, ID number RFMEFI57517X0164. 